\title{
DEFINIÇÃO AUTOMÁTICA DE ÁREAS CENSITÁRIAS NA FREGUESIA DE ALGÉS
}

\author{
Nelson Mileu ${ }^{1}$
}

\begin{abstract}
Resumo - Em Portugal, à semelhança de vários países, são efectuados recenseamentos decenais da população. Estes consistem, sinteticamente, numa fotografia do país onde estão descritas as principais características socio-económicas da população. Neste trabalho, assume-se que os censos constituem a metodologia de contagem populacional e, partindo dessa base, procura-se discutir a forma como a informação acerca dos indivíduos e alojamentos é geograficamente agregada. Neste sentido, apresenta-se a metodologia utilizada na freguesia de Algés para a definição automática de áreas censitárias, procurando que a geografia censitária proposta seja coerente e homogénea, resultando em áreas estatísticas mais flexíveis e comparáveis.
\end{abstract}

Palavras-chave: Censos, geografia censitária, representação geográfica, planeamento.

\begin{abstract}
Automatic statistical area definition in algés parish. Portugal, along with many other countries, conducts a population census every 10 years. This way of counting the population offers a detailed photography of the country where many socio-economic variables are captured. In this study, it is assumed that census will be the way of population data collection and therefore the discussion deals with the issue of geographical aggregation of individuals and households enumerated. In this context, a methodological proposal for an automatic census area creation in the parish of Algés is described. The minimum geographic unit for completing the aggregations is the building since it was the smallest and stable unit for this purpose. The building layer was obtained in the municipal GIS of Oeiras and is based on large scale cartography (1:1000). Mainly because the building layer is not contiguous, the study starts with the construction of a Thiessen polygon layer. This layer is drawn with the buildings layer centroids and having the master plan classes as barriers. The Thiessen polygon layer associated with the building attributes assumed a key role in this process and considering the range of possible data aggregation alternatives, the zone boundaries are drawn taking into account some principles of consistence and homogeneity. To achieve a measurement of homogeneity for the statistical areas the variables used were the number of households, floors and type of building (residential, commercial,
\end{abstract}

1 Geógrafo. E-mail: nmileu@municipia.pt 
both). The automatic statistical area definition deals with the aggregation of the basic geographic units (Thiessen polygon layer), seeking for new optimized areas, considering the characteristics of the building and having a reference number of households around 100. To demonstrate the methodology used for the automatic statistical area definition, three simulation results are presented in this article.

Key words : Census, census geography, geographic representation, planning.

Resumé - Définition automatique des unités spatiales de ReCENSEMEnT À algés, Les recensements de population sont effectués tous les dix ans au Portugal. Ils constituent une espèce de photographie du pays, où les principales caractéristiques socio-économiques de la population sont décrites. On discute la façon de découper spatialement l'information concernant les personnes et les logements. On présente la méthode qui a été utilisée dans la commune d'Algés pour définir automatiquement les unités spatiales de base, de façon à ce que la géographie censitaire résultante soit à la fois cohérente et homogène, facile à adapter et à comparer.

Mots-clés : Recensement, géographie censitaire, représentation géographique, aménagement.

\section{INTRODUÇÃO}

Os recenseamentos desempenham um papel fulcral para o desenvolvimento dos países, uma vez que permitem conhecer, além dos totais populacionais, a composição e a distribuição populacional, factores tão importantes em planeamento para se prever as necessidades de equipamentos ou infra-estruturas em domínios tão diversos como a saúde ou a educação, entre outros.

Este trabalho incide sobre um dos vectores fundamentais dos recenseamentos, a geografia censitária, e tem origem na investigação obtida numa dissertação de Mestrado em Sistemas de Informação Geográfica (SIG), apresentada em 2001 ao Instituto Superior Técnico.

Tem como objectivo a compreensão da problemática da representação geográfica de informação socio-económica e, mais concretamente, a apresentação de um quadro metodológico para a definição automática de áreas censitárias de forma coerente e enquadradas no apoio ao planeamento do uso do solo e gestão de infra-estruturas. Neste contexto, constróem-se de forma automática as fronteiras estatísticas que se consideram mais adequadas, procurando que a nova geografia censitária seja mais coerente e homogénea, resultando em áreas estatísticas mais flexíveis e comparáveis.

Como caso de estudo para a definição automática de áreas censitárias foi utilizada a freguesia de Algés, situada no concelho de Oeiras, devido às suas características predominantemente urbanas. No entanto, a metodologia aplicada é possível de ser utilizada noutros contextos, bastando, para tal, ajustar os critérios utilizados. 


\section{OS NOVOS DESAFIOS DA GEOGRAFIA CENSITÁRIA}

A forma e o conteúdo dos recenseamentos varia significativamente de país para país, sendo a sua representação geográfica constituída, na generalidade dos países, por pequenas áreas. Os princípios subjacentes a esta representação são a existência de homogeneidade e a não alteração do fenómeno dentro das fronteiras da área censitária.

As razões pelas quais a agregação de informação é a forma mais comum na manipulação de informação de natureza censitária prendem-se fundamentalmente com a redução do volume de informação, por motivos óbvios de confidencialidade dos dados e com a criação de padrões espaciais (Openshaw e Alvanides, 1996). Prefigurando-se como a solução mais consistente na representação geográfica da informação censitária e apesar de todos os cuidados inerentes a uma representação por mapas coropletos, esta acarreta alguns problemas, que residem no facto dos dados serem alterados, na perda de informação, na possibilidade de se acrescentar «ruído», na generalização e fundamentalmente na alteração de entidades (ou objectos).

Apesar dos totais e das estatísticas sumárias em cada unidade censitária parecerem correctos, a informação não pode ser interpretada de forma precisa. A questão chave é a de saber se as fronteiras das unidades censitárias fazem sentido em relação à representação de padrões micro-espaciais, uma vez que o problema da análise espacial reside fundamentalmente no facto da informação se referir a objectos que não são os mesmos, e por isso, não comparáveis.

Segundo Openshaw et al. (1998), os aspectos (segundo uma perspectiva geográfica) pelos quais a maioria das unidades censitárias não constituem entidades comparáveis devem-se:

- à não homogeneidade;

- à grande variação no tamanho populacional;

- à instabilidade no tempo;

- às diferenças em forma e tamanho físico;

- ao desenho informal ou incoerente e em desacordo com regras, mas sim de acordo com critérios de conveniência e de confidencialidade.

Devido à difusão generalizada dos SIG, e de uma forma quase repentina, os utilizadores depararam-se como uma nova instância do clássico problema da modelação de diferentes unidades do tipo área, o que para Openshaw e AlvaniDES (1996) apenas acentua a importância do Problema da Modificabilidade das Unidades Tipo Área (ProbMUTA) ${ }^{2}$.

2 Este problema é designado na literatura anglo-saxónica como modifiable areal unit problem e foi traduzido como Problema da Modificabilidade das Unidades Tipo Área (ProbMUTA). Constitui o centro de diversas investigações com o objectivo de determinar as suas consequências na análise espacial. É um problema endémico a todos os dados agregados espacialmente, podendo-se resumir na existência de diferentes resultados/conclusões perante várias reagregações geográficas dos mesmos dados base. 
Este problema específico da análise espacial assenta no facto de se poderem definir inúmeras unidades tipo área sem se saber qual a mais correcta (MARTIN, 1996; WrigLEY et al., 1996), mesmo com a imposição de tamanhos ou contiguidades, compreendendo dois problemas distintos, mas relacionados:

- a escala, que se relaciona com o número de zonas;

- e a agregação, que foca as diferentes opções existentes quanto ao esquema de fronteiras, tendo em conta o nível de agregação da informação.

O problema que agora se debate assenta no facto da mesma informação micro-espacial dar origem a diferentes representações espaciais, consoante a agregação efectuada, podendo-se facilmente manipular análises e conclusões. Se até há poucos anos os investigadores e planeadores viviam naturalmente com estes problemas, talvez condicionados pela indisponibilidade de informação, outros ignoravam-nos deliberadamente adulterando muitas análises e conclusões, esquecendo que as entidades agregadas não representam entidades comparáveis ou unidades naturais para representação de informação (CoOMBES, 1995).

Para Openshaw e Alvanides (1996), existem três soluções possíveis para a resolução do problema da modelação flexível de unidades do tipo área: a opção de não reagregar, a reagregação como opção explícita e as representações através de superfícies. Se a primeira opção é pouco razoável, uma vez que a reagregação de informação é um mal necessário, a última alternativa também não oferece muitas vantagens, uma vez que a informação de natureza socio-económica encontra-se, normalmente, agregada em unidades do tipo área. Segundo estes autores, a abordagem do tipo contínua não é a melhor, visto introduzir distorções e erros que impedem o seu relacionamento com a "realidade».

Em relação à segunda opção, podem apresentar-se várias alternativas para contornar os problemas até agora apresentados. No âmbito da definição automática de áreas importa, no entanto, distinguir dois processos (OpEnsHaw, 1996):

- o primeiro radica na identificação e definição de áreas que partilham características comuns (ex.: regionalização funcional, classificação espacial);

- o segundo processo consiste numa ferramenta de análise espacial onde se procura representar num mapa a interacção entre uma função (ou um valor) a optimizar e a informação a ser agregada.

Estas são formas distintas de abordar a agregação de informação e importa, no contexto da definição automática de áreas censitárias, explorar a segunda. A reagregação da informação das unidades estatísticas básicas, através de métodos de optimização, permite contornar o problema das unidades estatísticas definidas com base num objectivo principal que é a recolha de informação, constituindo unidades com deficiências ao nível da homogeneidade. Quando 
se utilizam as fronteiras estatísticas para cartografar uma variável censitária, há que ter em atenção que essas mesmas fronteiras são artificiais, devendo a sua utilização, com o intuito de representar variações espaciais, ser feita com alguma prudência. As unidades estatísticas tipo área, como as portuguesas, só são unidades «naturais» para o fim a que se destinam, ou seja, a recolha de informação.

A solução para o ProbMUTA passa pela sensibilização e reconhecimento dos problemas inerentes a toda a informação do tipo área e, sobretudo, pela construção das entidades de forma coerente e independente e ainda inseridas num determinado objectivo. O problema das unidades modificáveis do tipo área existirá sempre, importa é tê-lo em conta e paralelamente construir unidades estatísticas que sejam coerentes do ponto de vista da representação geográfica.

\section{CONCEITOS UTILIZADOS NA DEFINIÇÃO AUTOMÁTICA DE ÁREAS CENSITÁRIAS}

A definição automática de áreas censitárias assenta na conjugação de três fases que permitem operacionalizar a metodologia para a construção destas:

- a primeira incide na escolha da unidade mínima de agregação, com o intuito de definir as unidades básicas que permitirão construir as áreas censitárias;

- a segunda corresponde à definição dos critérios a utilizar e a sua transposição para o contexto do caso de estudo;

- a última consiste na operacionalização dos conceitos anteriores, através da implementação do algoritmo que permitiu programar a aplicação de suporte à definição automática das áreas censitárias.

\section{Unidades básicas de agregação geográfica}

A área de estudo corresponde à freguesia de Algés, pertencente ao concelho de Oeiras (fig. 1).

As unidades geográficas que, por agregação, permitirão definir as unidades estatísticas fundamentais foram construídas com base nos polígonos de Thiessen (fig. 2), estruturados a partir dos 1280 edifícios da freguesia de Algés. O tema dos edíficios foi disponibilizado a partir do SIG da Câmara Municipal de Oeiras e é suportado por cartografia digital à escala 1/1 000.

A escolha dos edifícios como unidade básica de agregação deve-se ao facto desta ser a mais pequena, estável e precisa unidade para este fim. A construção dos polígonos de Thiessen, a partir dos centróides dos edifícios, tem por objectivo a definição de uma superfície contígua, permitindo assim a definição automática das áreas estatísticas. 


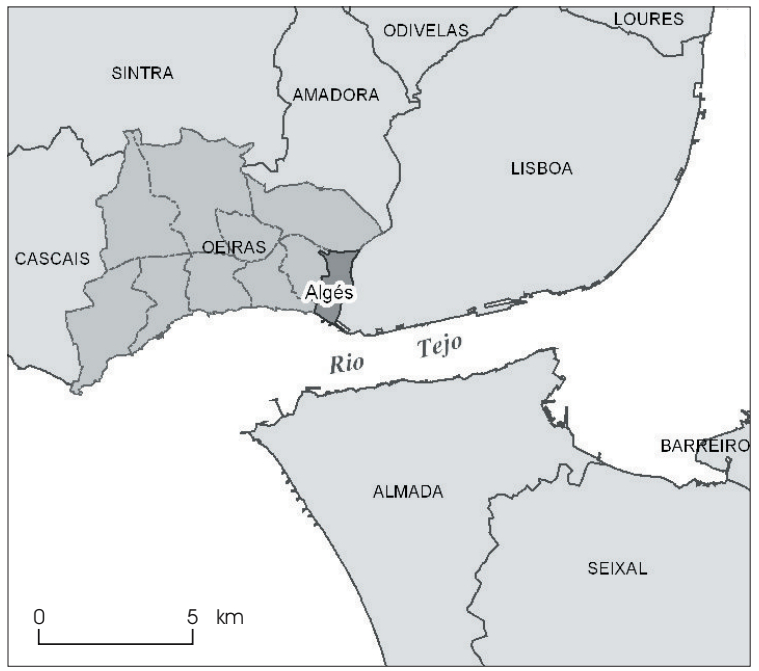

Fig. 1 - Localização da freguesia de Algés.

Fig. 1 - Geographical location of Algés parish.

Fig. 2 - Cobertura de polígonos de Thiessen resultante do tema edifícios (pontos) utilizando como restrição as classes de ordenamento do PDM.

Fig. 2 - Thiessen polygons resulting from building theme (points) using Master Plan classes as barriers.

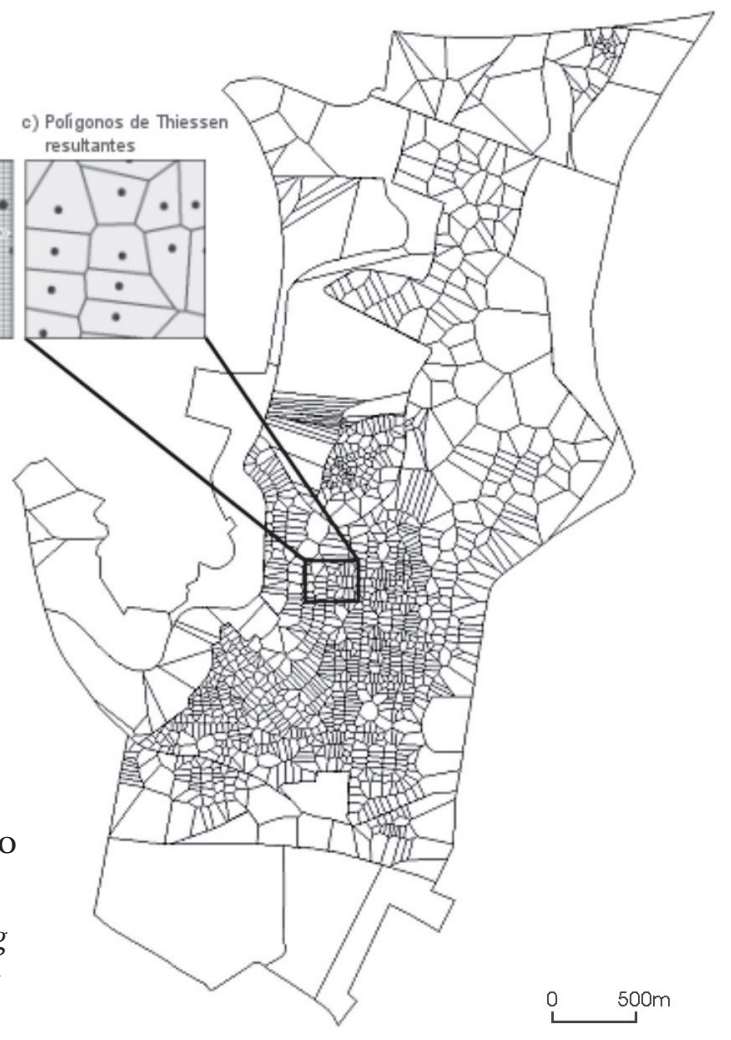


A criação dos polígonos de Thiessen foi suportada por um tema de pontos que correspondem aos centróides dos edifícios, tendo este herdado automaticamente os atributos presentes no tema dos edifícios. Após a criação do tema de centróides, geraram-se os polígonos de Thiessen, utilizando-se como barreiras os limites das classes de ordenamento definidas no Plano Director Municipal (PDM). A construção dos polígonos foi efectuada à custa de uma rotina, tendo-se obtido a cobertura de polígonos que constituiu a base geográfica de partida para a definição automática das áreas censitárias. Devido ao desfasamento de escalas entre o tema dos edifícios (1/1 000) e o PDM (1/10 000), houve necessidade de uma edição gráfica prévia que permitiu a compatibilização entre os dois temas nas situações de conflito.

Associado a cada edifício, encontra-se sempre presente o campo «CHAVE» (fig. 3). Este campo corresponde à concatenação do campo COD_RUA (código identificador do arruamento) com o campo NUM_POLICIA (número de polícia do edifício). Constitui um identificador unívoco do edifício que permite a qualquer tema estabelecer uma relação (1:1) com a tabela descritiva dos edifícios, tendo por essa razão, sido utilizado como identificador no processo de criação dos polígonos de Thiessen.

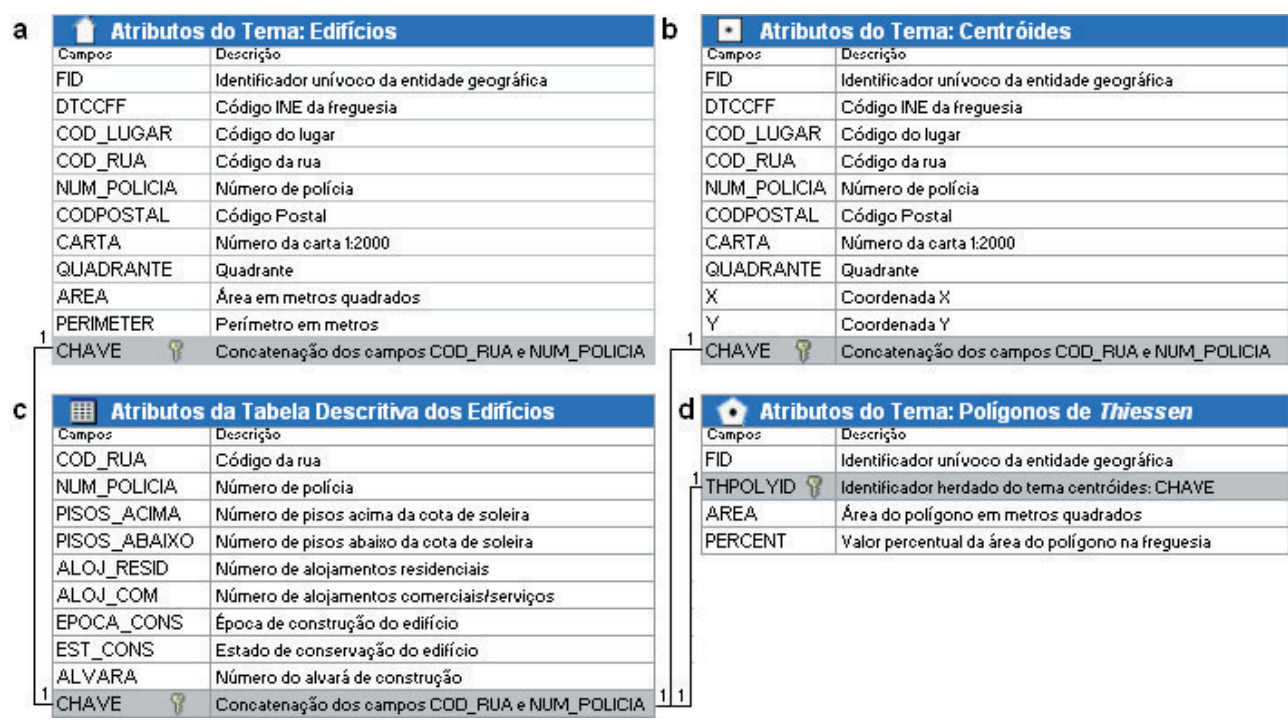

Fig. 3 - Estrutura das tabelas: atributos do tema edifícios (a), atributos do tema centróides (b), descrição dos edifícios (c) e atributos dos polígonos de Thiessen (d).

Fig. 3 - Tables design: buildings layer attributes (a), centroids layer attributes (b), buildings descriptive attributes (c) and Thiessen polygons layer attributes (d).

O processo de estruturação dos dados geográficos permitiu que os temas pudessem estabelecer uma relação com os atributos descritivos dos edifícios, acedendo-se desta forma às suas características bem como à sua topologia 
(número de alojamentos residenciais, comerciais e de serviços) ou o número de pisos. Estas variáveis assumem o papel central do esquema de classificação dos edifícios e do processo de definição de áreas homogéneas (Quadro I), desempenhando por essa razão uma função essencial na definição automática de áreas censitárias. Não obstante existirem outras variáveis de carácter urbanístico associadas ao tema de edifícios como o estado de conservação do edifício, o número do alvará de construção ou a época de construção, apenas esta última foi utilizada no processo de definição das áreas estatísticas.

\section{Os critérios de agregação}

A agregação de áreas ou a redefinição de fronteiras, especialmente políticas, não é um problema novo em geografia. Bem pelo contrário, desde a revolução quantitativa da geografia que, com maior ou menor entusiasmo, podemos encontrar diversos algoritmos e critérios que procuram resolver este tipo de problemas (Macmillan e Pierce, 1994; Openshaw, 1979).

Um dos critérios estabelecidos na definição automática de áreas estatísticas refere-se à sua construção de acordo com as zonas-plano definidas para a área de estudo. Desta forma simples, pode-se avaliar e controlar a evolução do processo socio-económico, apoiando com muito mais utilidade o processo de planeamento urbanístico e de gestão de infra-estruturas. A vantagem desta restrição pode ser facilmente entendida, se pensarmos em termos de políticas urbanas, uma vez que o planeamento se realiza usualmente a pensar no futuro, tentando canalizar o desenvolvimento nas direcções que foram delineadas. Neste contexto, pode ainda acrescentar-se, como refere Lово (1999: 40), que o planeamento terá que se antecipar aos fenómenos de evolução do povoamento e mobilizar os meios necessários para os influenciar no sentido desejável, racional, visando a sustentabilidade e a qualificação dos espaços urbanos e regionais pelo que uma construção das áreas estatísticas enquadrada com o planeamento constitui indubitavelmente, nesse contexto, uma ferramenta crucial.

O facto de se apoiar a definição de áreas estatísticas neste critério (espaços da mesma classe) vai permitir de uma forma simples uma definição mais coerente da área estatística, visto estar a envolver espaços que partilham, desde logo, algumas características socio-económicas e urbanísticas comuns. Por outro lado, alarga-se o prazo de vida das áreas estatísticas, controla-se o desenvolvimento a que cada uma das classes de espaço é sujeita e confere-se-lhe um propósito.

Outro critério utilizado para controlar os efeitos da agregação dos dados em áreas passa pela construção de níveis de agregação espacial que tenham em comum algumas propriedades. Estas passam, no essencial, por variáveis de carácter urbanístico, mormente o tipo de edifício ou mais especificamente a sua função e a sua inserção urbana. Os níveis de homogeneidade calculados com base nestes critérios deverão obviamente ser entendidos como uma forma de assegurar a coerência da área a definir, garantindo um controlo, mesmo que 
básico, sobre as diferenças geográficas. Por outro lado, qualquer tentativa de aprofundamento e diversificação da utilização de variáveis que controlem a heterogeneidade poderia causar alguns problemas, nomeadamente ao nível da compatibilização entre variáveis. Uma área homogénea deverá conter diversos elementos $(\mathrm{x})$, inseridos entre determinados limites $\left(\mathrm{Y}_{1}\right.$ e $\left.\mathrm{Y}_{2}\right)$, que variam de atributo para atributo:

$$
\mathrm{S}_{\mathrm{i}}=\left\{\mathrm{x} \mid \mathrm{Y}_{1}<\mathrm{Y}<\mathrm{Y}_{2}\right\}
$$

Para os ensaios efectuados e tendo em conta o contexto urbano do caso de estudo, considerou-se uma variável de controlo de homogeneidade que consistiu numa classificação dos edifícios. Inspirado na classificação de uso do solo de KAISER et al. (1995), apuraram-se as utilizações existentes na freguesia de Algés, associando aos diferentes usos o número de pisos como variável discriminatória das diferentes densidades (Quadro I).

Quadro I - Esquema de classificação dos edifícios.

Table I - Building classification model.

\begin{tabular}{|c|c|c|c|c|c|}
\hline \multicolumn{3}{|c|}{ Critérios } & 1. & Critério & 2. \\
\hline $\begin{array}{c}\text { N. }{ }^{\circ} \\
\text { Alojamentos } \\
\text { Residenciais }\end{array}$ & \multicolumn{2}{|r|}{$\begin{array}{c}\mathrm{N}^{\mathrm{o}} \\
\text { Alojamentos } \\
\text { Comerciais }\end{array}$} & $\begin{array}{c}\text { CLASSIFICAÇÃO } \\
\rightarrow\end{array}$ & N. ${ }^{\circ}$ Pisos & RECLASSIFICAÇÃO \\
\hline \multirow{2}{*}{$>0$} & \multirow{2}{*}{$\cap$} & \multirow{2}{*}{$=0$} & \multirow{2}{*}{ Exclusivamente Residencial } & $>2$ & $\begin{array}{c}\text { Residencial } \\
\text { (Alta Densidade) }\end{array}$ \\
\hline & & & & $₹=2$ & $\begin{array}{c}\text { Residencial } \\
\text { (Baixa Densidade) }\end{array}$ \\
\hline \multirow{2}{*}{$>0$} & \multirow{2}{*}{$\cap$} & \multirow{2}{*}{$>0$} & \multirow{2}{*}{ Misto (Habitação/Comércio) } & $>2$ & $\begin{array}{c}\text { Misto } \\
\text { (Alta Densidade) } \\
\end{array}$ \\
\hline & & & & $₹=2$ & $\begin{array}{c}\text { Misto } \\
\text { (Baixa Densidade) }\end{array}$ \\
\hline
\end{tabular}

O critério de dois pisos como fronteira entre os edifícios de baixa e de alta densidade justifica-se, em primeiro lugar, pela necessidade da separação estatística entre os edifícios cujo número de pisos era inferior a - $1 / 2$ desvio padrão da média e os restantes. Por outro lado, a distinção entre os edifícios de baixa e alta densidade efectuou-se desta forma simples, visto que permite distinguir entre edifícios do tipo vivenda ou construções de baixa altura e edifícios tipo prédio.

A esta classificação acrescentaram-se ainda os edifícios exclusivamente comerciais/serviços que, apesar de não entrarem para a contabilização do número de alojamentos, apresentam características específicas importantes para a definição de áreas homogéneas (fig. 4). 


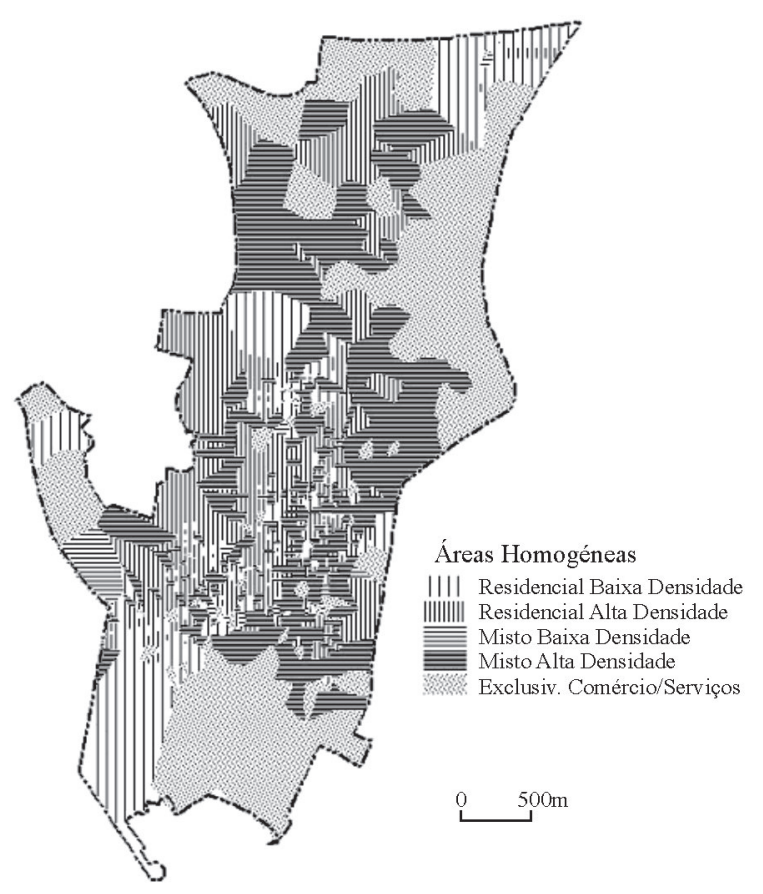

Fig. 4 - Áreas homogéneas segundo a classificação proposta.

Fig. 4 - Homogeneity results as proposed by the classification.

\section{O algoritmo para definição automática de áreas censitárias}

Para se definirem as áreas censitárias de forma automática, foi construída uma aplicação na linguagem Avenue do programa Arcview da ESRI que se denominou por DAAC - Definição Automática de Áreas Censitárias. O funcionamento do script que constitui o núcleo central da aplicação está resumido na figura 5.

O objectivo genérico do script é a construção automática de áreas, baseada num tamanho pré-estabelecido de alojamentos e em critérios de homogeneidade. Para que tal suceda, o primeiro passo do algoritmo consiste na escolha aleatória de uma área (A1) entre os $n$ polígonos de Thiessen que constituem a base geográfica de partida. Após esta escolha, os polígonos adjacentes (An) a A1 são seleccionados. Partindo desta selecção (An), o próximo passo consiste na escolha do polígono (A2) que será a área mais próxima em termos dos critérios de homogeneidade da área A1. A área (A2) que tiver as características mais próximas da área A1 é a escolhida para formar nova área (A1 + A2) que será sujeita a nova classificação. Após formar-se a nova área, o processo é reiniciado e só termina quando forem construídas as $n$ áreas. 


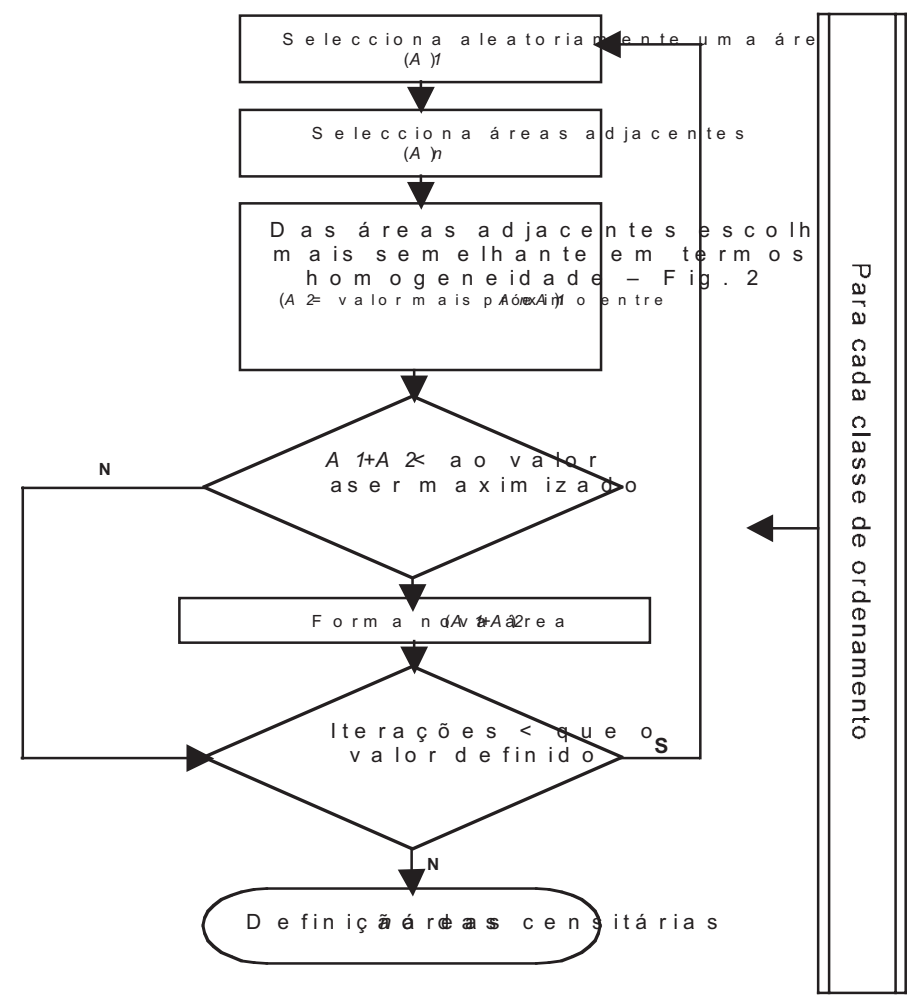

Fig. 5 - Algoritmo de funcionamento do script.

Fig. 5 - Script flowchart.

A esta escolha propositada da forma como os polígonos são classificados existe ainda uma importante restrição - o Plano Director Municipal. Pretende-se que as áreas construídas pertençam a espaços da mesma classe de ordenamento. Para que tal aconteça, foi construída uma rotina, que limita a definição das áreas aos espaços da mesma classe, forçando desta forma a dissolução de polígonos às mesmas características de ordenamento.

Os passos para a construção das áreas podem ser sinteticamente descritos da seguinte forma:

1) Escolha de uma área de estudo definida por uma unidade estatística fundamental e inviolável (Freguesia de Algés);

2) Criação da informação de suporte (Polígonos de Thiessen baseados numa cobertura pontual de edifícios);

3) Execução da aplicação DAAC:

3.1) Definição do número máximo de alojamentos por área censitária;

3.2) Para cada classe de ordenamento e com base nos critérios de homogeneidade pré-estabelecidos são definidas automaticamente $n$ áreas censitárias. 


\section{DEFINIÇÃO AUTOMÁTICA DE ÁREAS CENSITÁRIAS NA FREGUESIA DE ALGÉS}

A definição automática das áreas censitárias foi elaborada na freguesia de Algés, tendo-se efectuado para tal alguns ensaios que permitiram demonstrar a adequabilidade dos critérios e da aplicação. Dos vários ensaios efectuados, apresentam-se aqueles que permitiram demonstrar a exequibilidade da definição automática de áreas censitárias.

Nos três ensaios aqui apresentados, utilizou-se, como valor de referência máximo, 100 alojamentos residenciais por área estatística para a construção das áreas censitárias. Os resultados do primeiro ensaio corresponderam à criação de 111 áreas, tendo estas, em média, 88 alojamentos (desvio padrão de 28), atingindo um valor máximo de 98 alojamentos e um mínimo de 0 (fig. 6a).

Tendo presente a antecipação ao fenómeno de evolução do povoamento, acrescentou-se no ensaio seguinte outro nível de informação na construção de áreas estatísticas - os alvarás de loteamento. Na prática, a adição deste nível de informação resultou na reformulação dos polígonos para as novas localizações dos alojamentos com o consequente aumento do seu universo.

O acrescentar destes edifícios resultou na criação de 106 áreas, tendo estas em média 86 alojamentos (desvio padrão de 32), atingindo um valor máximo de 98 e um mínimo de 0 (fig. 6b).

O terceiro e último ensaio partiu da estrutura descrita anteriormente, acrescentando-se como objectivo, na construção das áreas estatísticas o facto dos polígonos a agregar pertencerem ao mesmo quarteirão.

Este ensaio (fig. 6c) deu origem a 120 áreas, tendo estas um valor médio de 74 alojamentos, atingindo um valor máximo de 100 e um mínimo de 0.

A validação dos ensaios foi suportada por uma variável de controlo que consistiu na época de construção do edifício. A utilização desta variável teve por objectivo verificar se agregações das áreas estatísticas, definidas de forma automática, têm uma variabilidade menor do que agregações efectuadas sobre as subsecções estatísticas utilizadas durante o recenseamento de 2001.

Este processo de validação consistiu na agregação das áreas por forma a que o número de alojamentos (200 alojamentos) por área estatística fosse semelhante para os dois métodos de definição das áreas estatísticas. Após a criação dos novos temas correspondentes à nova agregação das áreas, a validação foi efectuada à custa da comparação da variabilidade da época de construção (utilizando a codificação da tabela de atributos - fig. 3c) entre os dois processos de definição das áreas, podendo-se assim aferir para as novas agregações a sua estabilidade em relação à variável.

Refira-se que a escolha da variável consiste numa limitação do trabalho, pois o ideal seria a utilização de uma variável de natureza socio-económica e não de carácter urbanístico, uma vez que os critérios propostos para a definição de áreas homogéneas são urbanísticos. Esta limitação deriva essencialmente da impossibilidade de utilização de variáveis de carácter socio-económico com 
$\mathbf{a}$

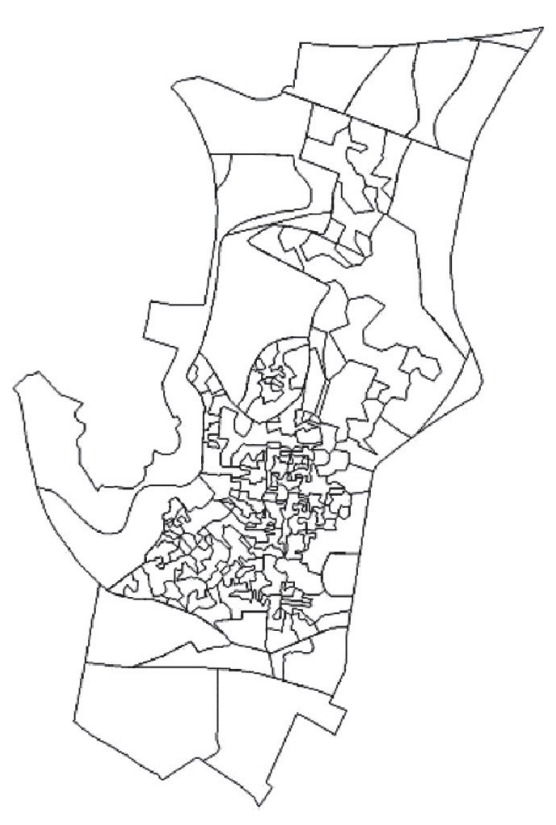

b

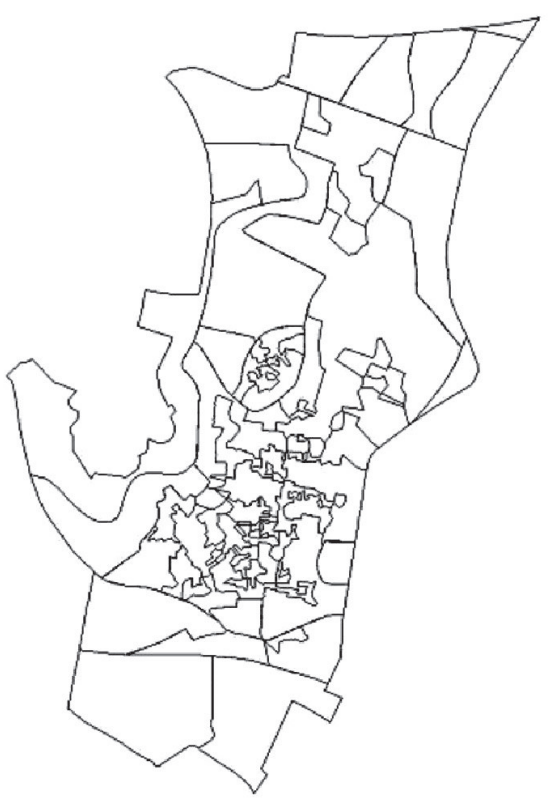

c

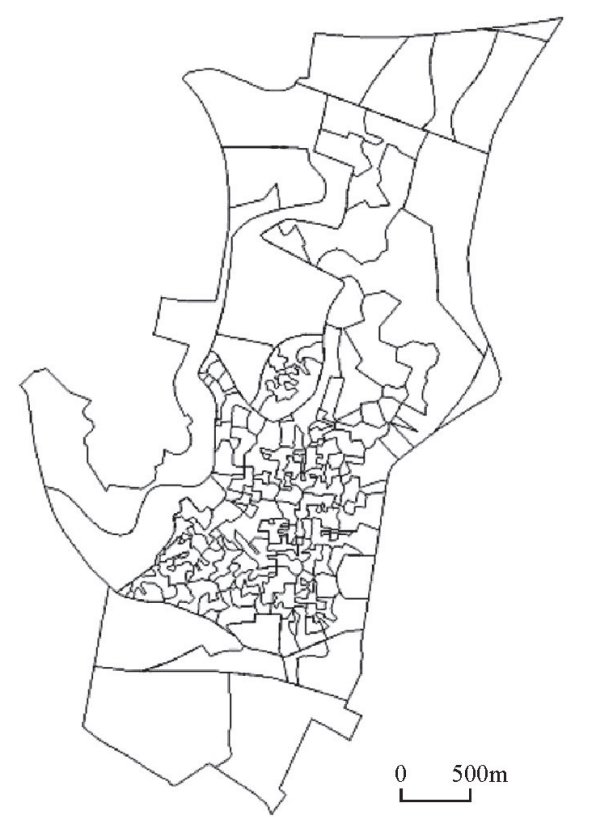

Fig. 6 - Resultados obtidos no $1 .^{\circ}$ ensaio (a), 2. ${ }^{\circ}$ ensaio (b) e 3..$^{\circ}$ ensaio (c).

Fig. 6 - First (a), second (b) and third simulation results (c). 
a desagregação do edifício, considerando-se por essa razão a variável utilizada uma alternativa.

A comparação da variabilidade da época de construção permitiu verificar que, para novas agregações efectuadas sobre os temas dos três ensaios, a variável época de construção tem em média características mais homogéneas do que a agregação efectuada sobre as áreas censitárias utilizadas no recenseamento de 2001, com especial destaque para o $3 .^{\circ}$ ensaio (Quadro II).

Quadro II - Média do desvio-padrão da variável 'época de construção dos edifícios' para novas agregações das áreas estatísticas

Table II - Building age average standard-deviation following new statistical areas aggregations

\begin{tabular}{|c|c|c|}
\hline & $\begin{array}{c}\text { Média dos } \sigma \\
\text { (áreas estatísticas) }\end{array}$ & $\begin{array}{c}\text { Média dos } \sigma \\
\text { (agregação de controlo } \\
\text { das áreas estatísticas) }\end{array}$ \\
\hline Subsecções (Censos 2001) & 1,39 & 1,69 \\
\hline 1. ${ }^{\circ}$ Ensaio & 1,34 & 1,56 \\
\hline 2. ${ }^{\circ}$ Ensaio & 1,35 & 1,56 \\
\hline 3. ${ }^{\circ}$ Ensaio & 1,22 & 1,49 \\
\hline
\end{tabular}

A forma sequencial como foram efectuados os ensaios permitiu, por um lado, afinar a proposta metodológica para a definição das área estatísticas e, por outro, rever e melhorar a aplicação. No entanto, deve reter-se, como principal ilação destes ensaios, a metodologia em que assentou a definição das áreas estatísticas e que permitiu a construção destas de uma forma coerente e objectiva.

\section{NOTAS FINAIS}

Com o desenvolvimento dos SIG na última década, a manipulação de informação censitária depara-se com novos desafios ao nível da representação geográfica. As tendências de utilização de informação geográfica apontam no sentido da flexibilização, ao invés da imposição de fronteiras estatísticas. Mas, para se poder obter uma maior flexibilidade na definição de áreas estatísticas, não basta agregar os dados consoante os diferentes objectivos. Os dados sobre os quais serão efectuadas análises e retiradas conclusões têm de obedecer a critérios de coerência e homogeneidade, resultando em áreas estatísticas mais flexíveis e comparáveis. Com a metodologia exposta, demonstrou-se que é possível construir de forma automática áreas estatísticas enquadradas nestes objectivos. 
Um dos aspectos metodológicos a destacar consiste na definição de áreas estatísticas de acordo com as classes de ordenamento do PDM. Porém, deve referir-se que este não é estático e as classes presentes não são definitivas. Estas estão sujeitas a reapreciações das disposições nele consagradas e, deste modo, a revisões periódicas. Nas revisões, estão naturalmente em causa as plantas de síntese e as delimitações das classes de ordenamento que se podem reflectir nas propostas de definição de áreas estatísticas assentes na metodologia descrita, pelo que deverá existir sempre alguma ponderação na utilização das figuras de plano, como critério na definição de áreas estatísticas.

Outro aspecto a referir consiste na imposição de um número máximo de alojamentos. Apesar de nos ensaios efectuados ter sido utilizado um valor de 100 alojamentos como referência, ficou demonstrado que é possível construir áreas tendo em conta outros critérios pré-definidos (critérios de homogeneidade). Note-se ainda que, nos diferentes ensaios efectuados, se verificou que as áreas construídas nunca são iguais, não obstante a imposição sistemática das restrições poder conduzir à definição das áreas nesse sentido.

A metodologia proposta para a freguesia de Algés pode ser optimizada, respondendo assim a novos objectivos, constituindo a reapreciação dos critérios e dos procedimentos de construção das áreas, aspectos a considerar em desenvolvimentos futuros. Outro aspecto a considerar em abordagens futuras passará pela validação das áreas estatísticas com recurso a variáveis socio-económicas e por uma avaliação qualitativa das unidades construídas, através de análises estatísticas espaciais que permitam aferir o valor das áreas estatísticas do ponto de vista da representação geográfica, nomeadamente face ao problema das unidades modificáveis do tipo área.

Em suma, tentaram identificar-se dois vectores de mudança no planeamento da geografia censitária, nomeadamente a separação entre o processo de recolha e difusão de informação, e a utilização de procedimentos automáticos na definição de áreas estatísticas associados a um aumento da flexibilidade de utilização das áreas. Sem apontar uma solução mágica para o problema das unidades modificáveis do tipo área, foram apresentados procedimentos inovadores face a estas questões que se baseiam na necessidade de determinar áreas sistema direccionadas a fins específicos. O objectivo consistiu na flexibilização da representação dos dados e na melhoria do desempenho do utilizador na determinação dessas áreas, adequando-as às particularidades do seu estudo.

\section{BIBLIOGRAFIA}

Coombes, M. (1995) - Dealing with census geography: Principles, practices and possibilities. In Openshaw, S. (ed.) - Census Users' Handbook, GeoInformation International, Cambridge: 111-132.

KAISER, E. et al. (1995) - Urban land use Planning, $4^{\text {th }}$ Edition. University of Illinois. 
Lово, M. (1999) - Planeamento regional e urbano. Universidade Aberta, Lisboa: 40.

Macmillan B. e Pierce, T. (1994) - Optimization modelling in a GIS framework: the problem of political redistricting. In Fotheringham, S. e Rogerson, P. (eds.) - Spatial Analysis and GIS, Taylor and Francis, London.

Martin, D. (1996) - Geographic information systems - Socioeconomic Applications, $2^{\text {nd }}$ Edition. Routlege, London.

Mileu, N. (2001) - Definição automática de áreas censitárias. Dissertação de Mestrado. IST, Lisboa.

Openshaw, S. (1979) - A Geographical solution to scale and aggregation problems in region-building, partitioning and spatial modelling. Transactions of the Institute of British Geographers, 2: 459-472.

Openshaw, S. (1996) - Developing GIS relevant zone based spatial analysis methods. In LoNGLEY, P. e BatTY, M. (eds.) - Spatial analyses: modelling in a GIS environment. GeoInformation International, Cambridge: 55-73.

Openshaw, S. e Alvanides, S. (1996) - Designing zoning systems for representation of socio-economic Data. Centre for Computational Geography, University of Leeds, Leeds.

Openshaw, S. et al. (1998) - Some further experiments with designing output areas for the 2001 UK census. Centre for Computational Geography, University of Leeds, Leeds.

Openshaw, S. e RaO, L. (1995) - Re-engineering 1991 census geography: serial and parallel algorithms for unconstrained zone design. Environment and Planning A, 27: 425-446.

Wrigley, N. et al. (1996) - Analysing, modelling, and resolving the ecological fallacy. In LoNGLEY, P. e BatTy, M. (eds.) - Spatial analyses: modelling in a GIS environment. GeoInformation International, Cambridge: 23-40. 